Published in final edited form as:

Sleep Med Clin. 2019 June ; 14(2): 177-184. doi:10.1016/j.jsmc.2019.01.009.

\title{
Cognitive Behavioral Therapy for Insomnia in Depression
}

\author{
Lauren D. Asarnow ${ }^{1}$ and Rachel Manber ${ }^{1}$ \\ ${ }^{1}$ Stanford University, Department of Psychiatry and Behavioral Sciences
}

\section{Keywords \\ depression; insomnia; comorbidity; cognitive behavioral therapy for insomnia}

The relationship between sleep and depression is bidirectional, complex, and apparent across the course of depression. Specifically, insomnia constitutes a risk for a future depressive episode among non-depressed individuals ${ }^{1}$ and its nocturnal symptoms are likely prodromal symptoms of depression among those who remitted from a depressive episode. ${ }^{2}$ During depressive episodes as many as $67-84 \%$ of adults and $57 \%$ of children and adolescents report difficulties initiating or maintaining sleep ${ }^{3-5}$ and more severe insomnia symptoms are independently associated with greater functional impairment ${ }^{6}$ and depressive symptom severity ${ }^{7}$. Persistence of insomnia symptoms is common among those with incomplete remission of depression $(94.6 \%)^{8}$ and even among those who fully remit following antidepressant therapy $(72 \%) ;{ }^{9}$ this suggests that treatment for depression does not provide sufficient sleep remedy. Furthermore, presence of residual poor sleep among those who remit from depression constitute a significant risk for a future depressive episode. ${ }^{10,11}$

The research reviewed in this manuscript highlights some of the progress made over the past five years that elucidates this complex relationship between depression and insomnia as well as challenges in the treatment of insomnia when comorbid with depression. Overall the data we review support the idea that insomnia may be instigating, maintaining, and exacerbating depression.

\section{Poor sleep prospectively predicts depression}

Longitudinal epidemiologic studies in adults found that disturbed sleep and insomnia increase the risk of a future episode of major depressive disorder one to three years later. For reviews, see Riemann and Voderholzer and Franzen. ${ }^{12,13}$ In a 2011 meta-analysis of 21

\footnotetext{
Please address correspondence concerning the manuscript to Lauren D. Asarnow, Ph.D., Stanford University, Department of Psychiatry and Behavioral Science 401 Quarry Road \#3342, Stanford, CA 943050, United States. Phone: (650) 736-4213. lasarnow@stanford.edu.

Disclosure Statement: The authors certify that we have no affiliations with or financial involvement within the past 5 years and foreseeable future (eg, employment, consultancies, honoraria, stock ownership or options, expert testimony, grants or patents received or pending, royalties) with any organization or entity with a financial interest in or financial conflict with the subject matter or materials discussed in the manuscript.

Publisher's Disclaimer: This is a PDF file of an unedited manuscript that has been accepted for publication. As a service to our customers we are providing this early version of the manuscript. The manuscript will undergo copyediting, typesetting, and review of the resulting proof before it is published in its final citable form. Please note that during the production process errors may be discovered which could affect the content, and all legal disclaimers that apply to the journal pertain.
} 
studies that prospectively evaluated poor sleep as a risk factor for a new depressive episode, Baglioni and colleagues concluded that presence of insomnia symptoms among adults without depression represents a significant risk for developing depression later. ${ }^{1}$ Four studies published since then are consistent with this conclusion. The first of these studies was a population-based sample of twins, which found that subjective poor sleep increased the risk of disability retirement due to depression 10-20 years later. ${ }^{14}$ The second was a large study of over 24,000 adults, using prospective data collected at two time points ten years apart. ${ }^{15}$ The study found a bidirectional relationship between depression and insomnia symptoms. ${ }^{15}$ The three other studies suggest that persistence of insomnia symptoms might be particularly relevant to the prediction of future depressive episodes. The first of these two studies was conducted among non-depressed adults in Korea. It found that persistence of insomnia symptoms (in this study defined as insomnia at 2 or more time points) significantly increased the rate in which depression occurred over 6 years. ${ }^{16}$ The second study focused on a sample of community dwelling older adults. It found that persistent sleep disturbance (defined as presence of insomnia symptoms across three time points during a year-long period) was associated with depression the following year. ${ }^{17}$ Lastly, a recent meta-analysis of 23 cross-sectional studies in older adults found that self-reported sleep disturbances increased the risk of the onset of depression (relative risk $[R R]=1.92$ ) and persistent sleep disturbances increased the risk of the development $(R R=3.90)$, recurrence $(R R=7.70)$, and worsening $(\mathrm{RR}=1.46)$ of depression in older adults. ${ }^{18}$

Research on poor sleep as a predictor of later depression in youth lagged behind the adult literature but increased in the past decade. Johnson, Roth $\&$ Breslau $^{19}$ found that, among youth age 13-16 with comorbid insomnia and depression, insomnia preceded depression in $69 \%$ of the sample; there was a significant association between prior insomnia and onset of depression even after adjusting for gender, race/ethnicity, and any prior anxiety disorder. ${ }^{19} \mathrm{~A}$ study of 289 twin pairs found that sleep problems at age 8 predicted depression at age 10, with some indication that this association might be due to genes. ${ }^{20} \mathrm{~A}$ recent review of the literature concluded that childhood sleep problems significantly predicted higher levels of depression, but not viceversa. ${ }^{21}$ More recently, utilizing data from the Great Smoky Mountains Study, authors found a bidirectional relationship between sleep and depression, such that sleep problems during childhood predicted increases in the prevalence of later depression and anxiety symptoms in adolescents and depression in childhood predicted increases in sleep problems over time. ${ }^{22}$ We examined specific sleep parameters that predict future depressive episodes using a nationally representative sample of adolescents. We found that late bedtime in middle school predicted more depression symptoms in young adulthood. 23

\section{Poor sleep prospectively predicts suicidality and self-harm:}

Results from a recent meta-analysis indicate that, among adults, insomnia is also a prospective risk factor for suicidal ideation, suicidal behavior, and completed suicide, even in studies that adjusted for the presence or absence of depressive disorder or symptoms. ${ }^{24} \mathrm{~A}$ later study similarly found that persistent insomnia (defined as the presence of insomnia at two or more time points) among individuals without a depression diagnosis at baseline increases the risk of suicidal thoughts across the span of six years. ${ }^{16}$ The research regarding 
the relationship between sleep and suicidality and self-harm in adolescents is consistent with the adult literature. Self-reported difficulties initiating or maintaining sleep at ages 12-14 significantly predicted suicidal thoughts and self-harm behaviors at ages $15-17 .^{25}$

\section{Poor sleep associated with more severe depression, including suicidality}

Depressive symptom severity is positively associated with insomnia symptom severity. For example, in the National Comorbidity Study- Revision data, the presence of insomnia symptoms (difficulty initiating or maintaining sleep determined using the Quick Inventory of Depressive Symptoms-Self Report) ${ }^{26}$ was associated with more severe depression symptoms. ${ }^{6}$ Depressive symptom severity is also positively associated objective indices of sleep disturbance. For example, among postpartum women, objective (actigraphic) measures of difficulties maintaining sleep (e.g., sleep fragmentation, sleep efficiency, and time awake time after sleep onset) were significantly correlated with depressive symptom severity. ${ }^{27}$ The association between depressive symptom severity and poor sleep has also been documented among youth. ${ }^{5}$

Poor sleep is also associated with elevation in specific symptoms of depression. For example, Emslie and colleagues ${ }^{5}$ found that among children and adolescents with depressive disorders, insomnia symptoms, which were present in over half the sample, were associated with greater severity of specific depressive symptoms, including fatigue, suicidal ideation, physical complaints, and concentration. Similarly among college students with depressive symptoms, those reporting sleep disturbance had more anxiety symptoms than those without sleep disturbance. ${ }^{28}$

\section{Poor sleep associated with suicidality in adults and youth:}

Controlling for depressive symptoms, poor subjective sleep quality at baseline was associated with increased risk for death by suicide at a 10 year follow-up in a populationbased community sample of older adults. ${ }^{29}$ In adults, two recent publications also examined the relationship between objective sleep measures derived from sleep laboratory polysomnography and suicidal ideation among adults with treatment- resistant depression. The first study found that greater nocturnal wakefulness, particularly in the early morning hours (e.g. during the 4:00 AM hour), was significantly associated with next-day suicidal thoughts adjusting for depression severity. ${ }^{30}$ Secondary analysis of the same data found that, independent of depression severity, suicidal ideation was associated with specific sleep architecture; specifically less NREM Stage 4 sleep and more time awake in the middle of the night across Bipolar and Unipolar Depression groups. ${ }^{31}$

In children and adolescents, recent research has extended past research ${ }^{32}$ documenting the connection between sleep and suicidal ideation and self-harm by examining a number of specific subjective sleep complaints (i.e., difficulty maintaining sleep, sleep difficulty bedtime variability, short sleep duration, non-restorative sleep). One study of over 600 school-aged children from the community found that significantly more children with selfharm behaviors reported subjective difficulty maintaining sleep after adjusting for symptoms of depression. ${ }^{33} \mathrm{~A}$ cross-sectional, national and representative sample consisting of over 75,000 students (grades 7-12) in Korea found that non-restorative sleep and short sleep 
duration were significantly associated with suicidal ideation in adolescents. ${ }^{34}$ In another study, using a distinct sample of Korean adolescents, weekend catch-up sleep duration (an indicator of insufficient weekday sleep) was associated with suicide attempts and self-injury. 35

\section{Poor sleep associated with poor response to depression treatments}

The body of research on the relationship between treatment response to both psychotherapy and psychopharmacological depression treatment and subjective and objective measures of sleep is growing. Subjective and objectively measured sleep disturbance predict attrition and remission rates as well as stability of psychopharmacological depression treatment response. 36-38 The literature also documents persistent insomnia symptoms as a risk factor for depression symptom recurrence following treatment with interpersonal psychotherapy, despite continued maintenance treatment. ${ }^{10}$ Another study reports that patients with objectively measured sleep abnormalities (early REM latency, low sleep efficiency and greater REM density) who were treated with interpersonal psychotherapy had worse remission rates than those with more typical sleep profiles. ${ }^{39}$ More recently, a pooled sample of over 700 adult outpatients with depression drawn from six clinical trials at the University of Pittsburgh between 1982 and 2001, examined the association between objectively measured sleep parameters (via polysomnography) and remission from pharmacological (imipramine, nortriptyline, fluoxetine, paroxetine, or bupropion) and/or psychological (interpersonal therapy or cognitive behavioral therapy) treatments of depression. ${ }^{40}$ The study found that prolonged sleep latency ( $>30$ minutes) and shorter sleep duration ( $<=6$ hours) alone or in combination with insomnia predicted increased risk of non-remission (remission defined as a Hamilton Depression Rating Scale score of $<=7$ ) and that patients with three or more baseline sleep disturbances were three times less likely to reach remission. ${ }^{40}$

Interestingly, recent research in children and adolescents points to differences in antidepressant treatment response in those with and without insomnia symptoms by age group. One study in 166 depressed adolescents treated with a 12-week course of sertraline, cognitive-behavioral therapy, or their combination found that across treatment groups, lower response and remission rates were associated with pre-treatment and ongoing sleep disturbance across treatment groups. ${ }^{41}$ Another study in both children and adolescents treated with fluoxetine found that while response rates were similar in those with or without insomnia symptoms, there was a significant difference by age group; among adolescents, those with insomnia symptoms were less likely to respond to fluoxetine than those without; while in children, the reverse was true, those with insomnia symptoms were more likely to respond to fluoxetine than those without insomnia. ${ }^{5}$ It is not clear why age moderated the association between disturbed sleep and baseline and response to fluoxetine. The authors suggest that this might be related to developmental differences in sleep architecture between depressed children and adolescents. ${ }^{5}$

\section{Therapeutic strategies}

There are effective pharmacological and non-pharmacological treatments for both insomnia and depression. The treatment of comorbid depression and insomnia involves clinical 
decision about which and in what order to introduce the treatments for the two disorders. We focus our discussion below on evidence concerning the order of treatments for the two disorders, leaving out the complex issues involved in selection of specific medications and specific psychotherapies. We note that research on the question of sequencing included mainly cognitive behavioral therapy for insomnia (CBT-I) and for depression (CBT-D) and interpersonal therapy for depression (IPT).

\section{Sequential Treatment}

One strategy for treating patients with insomnia-depression comorbidity is to treat one disorder at a time. As reviewed earlier, starting the sequence with depression treatment alone will likely leave many with unresolved insomnia symptoms. Since residual insomnia symptoms constitute a risk for relapse and recurrence, it important to treat them. ${ }^{42}$ Watanabe and colleagues ${ }^{43}$ provide preliminary promising results in a pilot study that evaluated the strategy of adding a brief (four sessions) behavioral therapy for insomnia to usual care of patients who did not fully remit with pharmacotherapy for depression and experienced residual insomnia symptoms. The study found significant improvement in insomnia and depression symptoms, with large effect sizes relative to treatment as usual (Number Needed to Treat $[\mathrm{NNT}]=2$ ). In another pilot study Ashworth and colleagues ${ }^{44}$ compared four sessions of face-to-face CBT-I to self-help CBT-I for patients experiencing residual insomnia symptoms after being treated with antidepressants for at least 6 weeks. The results indicate that those treated with face-to-face CBT-I experienced greater improvement in insomnia and depression severity. ${ }^{44}$ It will be important to follow these promising results with larger studies and longer follow ups that can evaluate whether these sequential strategies positively impact the course of depression over time by reducing the risk or shortening the time to relapse and recurrence.

Research on the strategy of first treating insomnia is hindered by ethical challenges, which is why studies evaluating the efficacy of this strategy included only patients with milder depression and were short term. ${ }^{11,45}$ One exception is a recent small study that compared how patients with dual diagnosis of insomnia and major depressive disorder responded to nine weeks of online treatments with either CBT-I $(n=22)$ or CBT-D $(n=21) .{ }^{46}$ Results indicated that online CBT-I was significantly more effective than depression treatment in improving insomnia (57\% versus $19 \%$ of treatment completers no longer met criteria for insomnia) and comparable in reducing depression (37\% versus $21 \%$ of completers no longer met depression criteria). ${ }^{46}$ These preliminary results cannot be interpreted both because of the small sample and importantly also because there is a differential rate of completion of the two online treatments. One controlled and several uncontrolled studies of group CBT-I also find significant improvements in both insomnia and depression at post treatment. Norell-Clarke and colleagues ${ }^{47}$ investigated the effects of group CBT-I on insomnia and depressive symptomatology in a comorbid sample through a randomized controlled trial. The authors found that CBT-I was more efficient than a control treatment in reducing insomnia severity and CBT-I was associated with a higher proportion of remission from both insomnia and depression diagnoses than a control treatment. Ong and colleagues reported that group CBT-I among patients seeking treatment for insomnia in a sleep center was 
equally effective for those with and without elevation in depressive symptom severity and led to modest reduction in depression severity and suicidal ideation. ${ }^{48}$

\section{Concomitant treatment}

Another strategy for the treatment of depression-insomnia comorbidity is to treat both disorders concomitantly. This strategy has the advantage of more rapid reduction of suffering because patients could achieve relief of depression insomnia earlier than they would with the sequential approach. Below we review results from studies that evaluated the concomitant treatment strategy examining the effects of this strategy on both disorders. Randomized controlled studies clearly indicate that combining antidepressant medications with CBT-I effectively alleviates insomnia symptoms in adults ${ }^{49,50}$ and adolescents. ${ }^{51}$ Results pertaining to the effects of the concomitant approach on depression outcomes have been mixed. In an earlier randomized controlled pilot study we found that combining escitalopram with CBT-I improved remission from depression relative to escitalopram plus active control therapy for insomnia among adults with comorbid insomnia and major depressive disorder ${ }^{49}$ However, our subsequent larger RCT, the Treatment of Insomnia in Depression (TRIAD), did not replicate the results from the pilot study. The TRIAD study randomized individuals to receive an antidepressant pharmacotherapy algorithm with either CBT-I or the same control therapy as in the pilot study and found no difference in depression remission. ${ }^{50}$ However, further analysis revealed that improvements in insomnia at week 6 of treatment mediated eventual remission from depression. ${ }^{50}$ Similarly, in a randomized controlled trial that compare escitalopram plus CBT-I to either CBT-I plus placebo pill, or escitalopram plus 4-session sleep hygiene control in adults with major depressive disorder and insomnia disorder. ${ }^{52}$ Carney and colleagues ${ }^{52}$ found that although all groups selfreported sleep improved, only the CBT-I groups improved on objective sleep, and sleep worsened in the group that received antidepressants plus sleep hygiene. While depression symptoms across all groups improved significantly from baseline to post-treatment, change in depression symptoms from baseline to post-treatment did not differ between groups.

Similar to negative findings in adults, a pilot RCT in adolescents also failed to show benefits of adding CBT-I to depression treatment. The study compared the combination of CBT-D plus CBT-I to CBT-D plus sleep hygiene control therapy for insomnia among adolescents with comorbid insomnia and depression and found no difference in rates of recovery from depression between the two conditions. ${ }^{51}$ However, when limiting the analysis to those who remitted from depression, the researchers found a trend for faster remission among those in the CBT-I condition. ${ }^{51}$

The concomitant strategy also has the potential to impact patients' tolerance of antidepressant medications. The TRIAD study reported that CBT-I participants reported greater frequency of medication side effects and higher maximum side effect than participants randomized to the control insomnia therapy. However, no participant discontinued the study due to antidepressant side effects. ${ }^{50}$ 


\section{Challenges treating insomnia comorbid with depression}

Although, as reviewed in the previous section, CBT-I is efficacious for the treatment of insomnia comorbid with depression, treating insomnia comorbid with depression presents some unique challenges. Indeed, among patients participating in group CBT-I in a sleep clinic those with elevated depressive symptom severity are more likely to discontinue treatment. ${ }^{48}$ Moreover, a study from Edinger and colleagues ${ }^{53}$ found that patients with comorbid depression and insomnia who experienced the first onset of both disorders in childhood are less responsive to CBT-I than are those with their first onset as an adult. Also, the literature suggests that individuals with insomnia comorbid with depression may be at increased risk for poor adherence and response. We review below some of these risks and suggest strategies for addressing the challenges that they present when implementing CBT-I.

\section{Cognitions:}

Research indicates that patients with insomnia and depression report more unhelpful beliefs about sleep than those who experience insomnia without comorbid depression ${ }^{54}$ and that such beliefs are linked to more treatment resistant insomnia. ${ }^{55-57}$ Maladaptive beliefs about sleep might also serve as barriers to engagement and adherence to some of treatment recommendations. ${ }^{58}$ For example, a belief that $8 \mathrm{~h}$ of sleep is required in order to function can lead to increased anxiety and low engagement and/or adherence when the recommended time in bed is restricted to below the desired amount. Rumination, which is common in depression, ${ }^{59}$ is associated with worse sleep quality 60 and tendency to ruminate about daytime consequences of insomnia. ${ }^{60}$ Maladaptive beliefs and rumination that are common in depression can be addressed using cognitive therapy.

\section{Anhedonia:}

Some individuals with depression find it difficult to make the behavioral changes necessary for improving their sleep. They go to bed earlier then when euthymic, get out of bed later, or both because they do not enjoy activities that were previously pleasurable or rewarding and/or as an escape from emotional suffering. ${ }^{61}$ This makes it difficult to adhere to core treatment recommendations, such as sleep restriction ${ }^{62}$ and stimulus control instructions. ${ }^{63}$ Behavioral activation ${ }^{64}$ can be used to overcome these barriers to adherence. Behavioral activation involves helping depressed people reengage in their lives through focused activation strategies which counter patterns of avoidance, withdrawal, and inactivity that may exacerbate depressive episodes replacing them with opportunities to help individuals approach and access sources of positive reinforcement in their lives, which can serve a natural antidepressant function; it is an integral component to CBT-D. In the context of insomnia, behavioral activation is focused primarily on pre-bedtime behavior to facilitate postponing bedtime and in the morning to facilities getting out of bed at the recommended time.

\section{Conclusion}

In conclusion, the relationship between sleep problems and depression is complex, likely bidirectional and impactful. The adult and adolescent literature points to the presence of 
insomnia symptoms as an important predictor and/or precursor to a depressive episode. Moreover, insomnia symptoms are associated with more severe depression symptoms, including increased suicidality and predict poor depression treatment response in adults and adolescents. Fortunately, CBT-I is an effective, short-term treatment for insomnia symptoms when depression and insomnia symptoms are co-occurring. In the past five years research has taken two approaches to treating comorbid insomnia and depression symptoms; a sequential approach, where providers treat one disorder at a time, or a concomitant approach where both disorders are treated simultaneously. While initial results from studies that assessed the sequential approach (treating either insomnia prior to depression treatment or treating residual insomnia symptoms following depression treatment) showed promising results, more adequately powered randomized control trials with long-term follow-up are needed to evaluate how sequential strategies impact the course of depression over time. The literature on whether concomitant treatment of depression and insomnia results in improved depression outcomes is also in its nascent stages, with mixed results. Nonetheless, a recent finding that response to insomnia treatment mediated eventual remission from depression suggests that focused effort to improve insomnia has the potential to enhance depression treatment outcomes. To further improve the lives of patients with depression who experience insomnia it will be important to investigate which patients will do better in a sequential versus concomitant approach.

\section{References}

1. Baglioni C, Battagliese G, Feige B, et al. Insomnia as a predictor of depression: a metaanalytic evaluation of longitudinal epidemiological studies. J Affect Disord. 2011;135(1-3):10-19. [PubMed: 21300408]

2. Perlis ML, Giles DE, Buysse DJ, Tu X, Kupfer DJ. Self-reported sleep disturbance as a prodromal symptom in recurrent depression. J Affect Disord. 1997;42(2-3):209-212. [PubMed: 9105962]

3. Hamilton M Frequency of symptoms in melancholia (depressive illness). Br J Psychiatry. 1989;154:201-206. [PubMed: 2775946]

4. Ford DE, Kamerow DB. Epidemiologic study of sleep disturbances and psychiatric disorders. An opportunity for prevention? JAMA. 1989;262(11):1479-1484. [PubMed: 2769898]

5. Emslie GJ, Kennard BD, Mayes TL, et al. Insomnia Moderates Outcome of Serotonin-Selective Reuptake Inhibitor Treatment in Depressed Youth. Journal of Child and Adolescent Psychopharmacology. 2012.

6. Soehner AM, Kaplan KA, Harvey AG. Prevalence and clinical correlates of co-occurring insomnia and hypersomnia symptoms in depression. Journal of Affective Disorders. 2014.

7. Taylor DJ, Lichstein KL, Durrence HH, Reidel BW, Bush AJ. Epidemiology of insomnia, depression, and anxiety. Sleep. 2005.

8. McClintock SM, Husain MM, Wisniewski SR, et al. Residual symptoms in depressed outpatients who respond by $50 \%$ but do not remit to antidepressant medication. Journal of Clinical Psychopharmacology. 2011.

9. Nierenberg AA, Husain MM, Trivedi MH, et al. Residual symptoms after remission of major depressive disorder with citalopram and risk of relapse: A STARßD report. Psychological Medicine. 2010 .

10. Dombrovski AY, Cyranowski JM, Mulsant BH, et al. Which symptoms predict recurrence of depression in women treated with maintenance interpersonal psychotherapy? Depress Anxiety. 2008;25(12):1060-1066. [PubMed: 18781665]

11. Taylor DJ, Walters HM, Vittengl JR, Krebaum S, Jarrett RB. Which depressive symptoms remain after response to cognitive therapy of depression and predict relapse and recurrence? Journal of Affective Disorders. 2010. 
12. Franzen PL, Buysse DJ. Sleep disturbances and depression: risk relationships for subsequent depression and therapeutic implications. Dialogues Clin Neurosci. 2008;10(4):473-481. [PubMed: 19170404]

13. Riemann D, Voderholzer U. Primary insomnia: A risk factor to develop depression? Journal of Affective Disorders. 2003.

14. Paunio T, Korhonen T, Hublin C, et al. Poor sleep predicts symptoms of depression and disability retirement due to depression. Journal of Affective Disorders. 2015.

15. Sivertsen B, Salo P, Mykletun A, et al. The bidirectional association between depression and insomnia: The HUNT study. Psychosomatic Medicine. 2012.

16. Suh S, Kim H, Yang H-C, Cho ER, Lee SK, Shin C. Longitudinal Course of Depression Scores with and without Insomnia in Non-Depressed Individuals: A 6-Year Follow-Up Longitudinal Study in a Korean Cohort. Sleep. 2013.

17. Lee E, Cho HJ, Olmstead R, Levin MJ, Oxman MN, Irwin MR. Persistent Sleep Disturbance: A Risk Factor for Recurrent Depression in Community-Dwelling Older Adults. SLEEP. 2013.

18. Bao YP, Han Y, Ma J, et al. Cooccurrence and bidirectional prediction of sleep disturbances and depression in older adults: meta-analysis and systematic review. Elsevier.

19. Johnson EO, Roth T, Breslau N. The association of insomnia with anxiety disorders and depression: Exploration of the direction of risk. Journal of Psychiatric Research. 2006.

20. Gregory AM, Rijsdijk FV, Lau JYF, Dahl RE, Eley TC. The direction of longitudinal associations between sleep problems and depression symptoms: A study of twins aged 8 and 10 years. Sleep. 2009.

21. Alvaro PK, Roberts RM, Harris JK. A Systematic Review Assessing Bidirectionality between Sleep Disturbances, Anxiety, and Depression. Sleep. 2013.

22. Shanahan L, Copeland WE, Angold A, Bondy CL, Costello EJ. Sleep problems predict and are predicted by generalized anxiety/depression and oppositional defiant disorder. Journal of the American Academy of Child and Adolescent Psychiatry. 2014.

23. Asarnow LD, McGlinchey E, Harvey AG. The effects of bedtime and sleep duration on academic and emotional outcomes in a nationally representative sample of adolescents. Journal of Adolescent Health. 2014.

24. Pigeon WR, Pinquart M, Conner K. Meta-analysis of sleep disturbance and suicidal thoughts and behaviors. In:2012.

25. Wong MM, Brower KJ, Zucker RA. Sleep problems, suicidal ideation, and self-harm behaviors in adolescence. Journal of Psychiatric Research. 2011.

26. Manber R, Blasey C, Arnow B, et al. Assessing insomnia severity in depression: comparison of depression rating scales and sleep diaries. J Psychiatr Res. 2005;39(5):481-488. [PubMed: 15992557]

27. Park EM, Meltzer-Brody S, Stickgold R. Poor sleep maintenance and subjective sleep quality are associated with postpartum maternal depression symptom severity. Archives of Women's Mental Health. 2013.

28. Nyer M, Farabaugh A, Fehling K, et al. Relationship between sleep disturbance and depression, anxiety, and functioning in college students. Depression and Anxiety. 2013.

29. Bernert RA, Turvey CL, Conwell Y, Joiner TE. Association of poor subjective sleep quality with risk for death by suicide during a 10-year period a longitudinal, population-based study of late life. JAMA Psychiatry. 2014.

30. Ballard ED, Vande Voort JL, Bernert RA, et al. Nocturnal wakefulness is associated with next-day suicidal ideation in major depressive disorder and bipolar disorder. Journal of Clinical Psychiatry. 2016.

31. Bernert RA, Luckenbaugh DA, Duncan WC, Iwata NG, Ballard ED, Zarate CA. Sleep architecture parameters as a putative biomarker of suicidal ideation in treatment-resistant depression. Journal of Affective Disorders. 2017.

32. Goldstein TR, Bridge JA, Brent DA. Sleep Disturbance Preceding Completed Suicide in Adolescents. Journal of Consulting and Clinical Psychology. 2008.

33. Singareddy R, Krishnamurthy VB, Vgontzas AN, et al. Subjective and objective sleep and selfharm behaviors in young children: A general population study. Psychiatry Research. 2013. 
34. Park JH, Yoo JH, Kim SH. Associations between non-restorative sleep, short sleep duration and suicidality: Findings from a representative sample of Korean adolescents. Psychiatry and Clinical Neurosciences. 2013.

35. Kang SG, Lee YJ, Kim SJ, et al. Weekend catch-up sleep is independently associated with suicide attempts and self-injury in Korean adolescents. Comprehensive Psychiatry. 2014.

36. Buysse DJ, Reynolds CF, Houck PR, et al. Does lorazepam impair the antidepressant response to nortriptyline and psychotherapy? Journal of Clinical Psychiatry. 1997.

37. Dew MA, Reynolds CF, Houck PR, et al. Temporal profiles of the course of depression during treatment: Predictors of pathways toward recovery in the elderly. Archives of General Psychiatry. 1997.

38. Thase ME. Depression, sleep, and antidepressants. In:1998.

39. Thase ME, Fasiczka AL, Berman SR, Simons AD, Reynolds CF. Electroencephalographic sleep profiles before and after cognitive behavior therapy of depression. Archives of General Psychiatry. 1998.

40. Troxel WM, Kupfer DJ, Reynolds CF, et al. Insomnia and objectively measured sleep disturbances predict treatment outcome in depressed patients treated with psychotherapy or psychotherapypharmacotherapy combinations. Journal of Clinical Psychiatry. 2012.

41. Manglick M, Rajaratnam SM, Taffe J, Tonge B, Melvin G. Persistent sleep disturbance is associated with treatment response in adolescents with depression. Australian and New Zealand Journal of Psychiatry. 2013.

42. Kurian BT, Greer TL, Trivedi MH. Strategies to enhance the therapeutic efficacy of antidepressants: Targeting residual symptoms. In:2009.

43. Watanabe N, Furukawa TA, Shimodera S, et al. Brief behavioral therapy for refractory insomnia in residual depression: An assessor-blind, randomized controlled trial. Journal of Clinical Psychiatry. 2011.

44. Ashworth DK, Sletten TL, Junge M, et al. A randomized controlled trial of cognitive behavioral therapy for insomnia: An effective treatment for comorbid insomnia and depression. Journal of Counseling Psychology. 2015.

45. Manber R, Bernert RA, Suh S, Nowakowski S, Siebern AT, Ong JC. CBT for insomnia in patients with high and low depressive symptom severity: Adherence and clinical outcomes. Journal of Clinical Sleep Medicine. 2011.

46. Blom K, Jernelöv S, Kraepelien M, et al. Internet Treatment Addressing either Insomnia or Depression, for Patients with both Diagnoses: A Randomized Trial. Sleep. 2015.

47. Norell-Clarke A, Jansson-Fröjmark M, Tillfors M, Holländare F, Engström I. Group cognitive behavioural therapy for insomnia: Effects on sleep and depressive symptomatology in a sample with comorbidity. Behaviour Research and Therapy. 2015.

48. Ong JC, Kuo TF, Manber R. Who is at risk for dropout from group cognitive-behavior therapy for insomnia? Journal of Psychosomatic Research. 2008.

49. Manber R, Edinger JD, Gress JL, San Pedro-Salcedo MG, Kuo TF, Kalista T. Cognitive behavioral therapy for insomnia enhances depression outcome in patients with comorbid major depressive disorder and insomnia. Sleep. 2008.

50. Manber R, Buysse DJ, Edinger J, et al. Efficacy of Cognitive-Behavioral Therapy for Insomnia Combined With Antidepressant Pharmacotherapy in Patients With Comorbid Depression and Insomnia: A Randomized Controlled Trial. J Clin Psychiatry. 2016;77(10):e1316-e1323. [PubMed: 27788313]

51. Clarke G, McGlinchey EL, Hein K, et al. Cognitive-behavioral treatment of insomnia and depression inadolescents: A pilot randomized trial. Behaviour Research and Therapy. 2015.

52. Carney CE, Edinger JD, Kuchibhatla M, et al. Cognitive Behavioral Insomnia Therapy for Those With Insomnia and Depression: A Randomized Controlled Clinical Trial. Sleep. 2017;40(4).

53. Edinger JD, Manber R, Buysse DJ, et al. Are patients with childhood onset of insomnia and depression more difficult to treat than are those with adult onsets of these disorders? A report from the TRIAD study. Journal of Clinical Sleep Medicine. 2017.

54. Carney CE, Edinger JD, Manber R, Garson C, Segal ZV. Beliefs about sleep in disorders characterized by sleep and mood disturbance. Journal of Psychosomatic Research. 2007. 
55. Morin CM, Blais F, Savard J. Are changes in beliefs and attitudes about sleep related to sleep improvements in the treatment of insomnia? Behaviour Research and Therapy. 2002.

56. Tremblay V, Savard J, Ivers H. Predictors of the Effect of Cognitive Behavioral Therapy for Chronic Insomnia Comorbid With Breast Cancer. Journal of Consulting and Clinical Psychology. 2009.

57. Cvengros JA, Crawford MR, Manber R, Ong JC. The Relationship Between Beliefs About Sleep and Adherence to Behavioral Treatment Combined With Meditation for Insomnia. Behavioral Sleep Medicine. 2015.

58. Carney CE, Edinger JD. Identifying critical beliefs about sleep in primary insomnia. Sleep. 2006.

59. Thomsen DK, Mehlsen MY, Christensen S, Zachariae R. Rumination - Relationship with negative mood and sleep quality. Personality and Individual Differences. 2003.

60. Carney CE, Edinger JD, Meyer B, Lindman L, Istre T. Symptom-focused rumination and sleep disturbance. Behavioral Sleep Medicine. 2006.

61. Hauri P, Fisher J. Persistent psychophysiologic (learned) insomnia. Sleep. 1986.

62. Spielman AJJ, Saskin P, Thorpy MJJ. Treatment of chronic insomnia by restriction of time in bed. Sleep. 1987.

63. Bootzin RR, Epstein D, Wood JM. Stimulus control instructions. Hauri P Case studies in insomnia. 1991.

64. Jacobson NS, Martell CR, Dimidjian S. Behavioral Activation Treatment for Depression: Returning to Contextual Roots. Clinical Psychology: Science and Practice. 2006. 


\section{Synopsis:}

The authors sought to review the literature on Cognitive Behavioral Therapy for Insomnia in adults and adolescents with depression. Recent research has expanded upon previous research which established that sleep problems are an important predictor of depression, and that sleep problems are associated with more severe depression, more suicidality and worse depression treatment outcomes. The relationship between sleep problems and depression is complex, likely bidirectional and impactful. To further improve the lives of patients with depression who experience insomnia it will be important to investigate which patients will do better in a sequential versus concomitant approach. 